\title{
Plasma levels of lysophosphatidic acid in ovarian cancer versus controls: a meta-analysis
}

\author{
Yi-Yang Li ${ }^{1}$, Wen-Chao Zhang ${ }^{2}$, Jia-Ling Zhang ${ }^{3}$, Chang-Jun Zheng ${ }^{3}$, He Zhu ${ }^{3}$, Hui-Mei Yu ${ }^{4}$ and Li-Mei Fan ${ }^{3 *}$
}

\begin{abstract}
Background: In this study, using a meta-analysis approach, we examined the correlation between serum levels of lysophosphastidic acid (LPA) and ovarian cancer (OC).

Methods: Relevant published studies were identified from multiple scientific literature databases by using a pre-determined electronic and manual search strategy. The search results were screened through a multi-step process to select high-quality case-control studies suitable for the present meta-analysis. Mean values and standardized mean differences (SMD) were calculated for plasma LPA levels. Two investigators independently extracted the data from the studies and performed data analysis using STATA software version 12.0 (Stata Corp, College Station, TX, USA).

Results: Nineteen case-control studies met our selection criteria and contained a total of 980 OC patients, 872 benign controls and 668 healthy controls. Our meta-analysis results revealed that the plasma levels of LPA in OC patients were significantly higher than benign controls $(\mathrm{SMD}=2.36,95 \% \mathrm{Cl}: 1.61-3.10, P<0.001)$ and healthy controls $(\mathrm{SMD}=2.32$, $95 \%$ Cl: 1.77-2.87, $P<0.001$ ). Subgroup analysis by ethnicity showed that the plasma LPA levels in OC patients were significantly higher than the benign controls only in Asian populations ( $\mathrm{SMD}=2.52,95 \% \mathrm{Cl}$ : 1.79-3.25, $P<0.001$ ). However, a comparison between healthy controls and OC patients revealed that, in both Asians and Caucasians, the OC patients displayed significantly higher plasma LPA levels compared to healthy controls (all $P<0.05$ ).

Conclusion: Our meta-analysis showed strong evidence that a significantly higher plasma LPA levels are present in OC patients, compared to benign controls and healthy controls, and plasma LPA levels may be used as a biomarker or target of OC.
\end{abstract}

Keywords: Lysophosphatidic acid, Ovarian cancer, Bioactive phospholipid, Meta-analysis

\section{Introduction}

Ovarian cancer (OC) is the deadliest among gynecological cancers, with 5-year survival rates ranging between $30-50 \%$. OC is not a single disease but is a collection of diverse tumors with distinct morphologies and genetic deficiencies. The pathogenesis of OC is not well characterized, but most OCs occurs spontaneously, with only $5-10 \%$ of the cases linked to a genetic predisposition. According to the latest estimates, $\mathrm{OC}$ is the $7^{\text {th }}$ most common cancer worldwide, and the age-standardized incidence rates range from more than 11 per 100,000 women in central and eastern Europe to less than 5 per 100,000 in parts of Africa, and is the eighth most

\footnotetext{
*Correspondence: fanlimeiflm85@126.com

${ }^{3}$ Department of Gynaecology and Obstetrics, the Second Hospital of Jilin University, Ziqiang Street NO. 218, Nanguan District, Changchun 130041, People's Republic of China

Full list of author information is available at the end of the article
}

common cause of cancer death in women globally [1]. OC often has no overt symptoms at early stages and the disease is usually advanced at diagnosis, with metastatic spread, which is the main reason for the high death rates associated with $\mathrm{OC}$ [2]. The late diagnosis and advanced metastatic stage severely limits treatment options and severely impacts the quality of life in OC patients [3]. Although most OC patients with an advanced disease initially respond to first-line therapy, only 10-15\% will maintain a complete response. Therefore, discovery of underlying mechanisms and disease factors promoting tumor growth and metastasis is of urgent need for developing novel tools for OC diagnosis and treatment [4].

LPA is a small bioactive phospholipid present in ascetic fluid and blood of OC patients [4]. The G

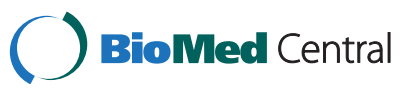

(C) 2015 Li et al. This is an Open Access article distributed under the terms of the Creative Commons Attribution License (http://creativecommons.org/licenses/by/4.0), which permits unrestricted use, distribution, and reproduction in any medium, provided the original work is properly credited. The Creative Commons Public Domain Dedication waiver (http:// creativecommons.org/publicdomain/zero/1.0/) applies to the data made available in this article, unless otherwise stated. 
protein-coupled receptors of the endothelial differentiation gene (Edg) family are stimulated by LPA and LPA mediated signaling effects cell proliferation, invasion, smooth muscle cell contraction, platelet aggregation, cell migration, cell survival, wound healing and alteration in morphology and differentiation of cells [5-7]. LPA mediated pathways are prominently linked to tumor growth and metastasis in various cancers and thus significant efforts are underway to understand the precise role of LPA and design effective intervention strategies. LPA is converted from lysophospholipids in the serum and plasma, and from phosphatidic acid in platelets and cancer cells [8]. LPA production from lysophospholipids requires the action of phospholipase A1 (PLA1)/PLA2 plus lysophospholipase D (lysoPLD), while the production of LPA from phosphatidic acid requires phospholipase D (PLD) plus PLA1/PLA2 activities $[8,9]$. Previous studies showed that ovarian tumor cells are a major source of LPA and autotaxin (ATX)/lysophospholipase $\mathrm{D}$ (PLD) pathway is the primary LPA producing pathway in ovarian tumor cells [10]. Consistent with this, plasma levels of LPA are strongly associated with presence of ovarian tumors and plasma levels of LPA are significantly higher in $\mathrm{OC}$ patients compared to benign ovarian lesions [4, 11]. Furthermore, previous studies showed that increased LPA levels are closely associated with the elevated expression levels of other prominent metastasis promoters critical for OC progression $[12,13]$. Nevertheless, perhaps due to the complexity of the LPA pathway and the diversity of its receptors, several other studies reported results contradicting the links between LPA and ovarian cancer $[14,15]$. Therefore, we performed a meta-analysis to closely examine this issue and obtain a correlation between plasma LPA level and OC.

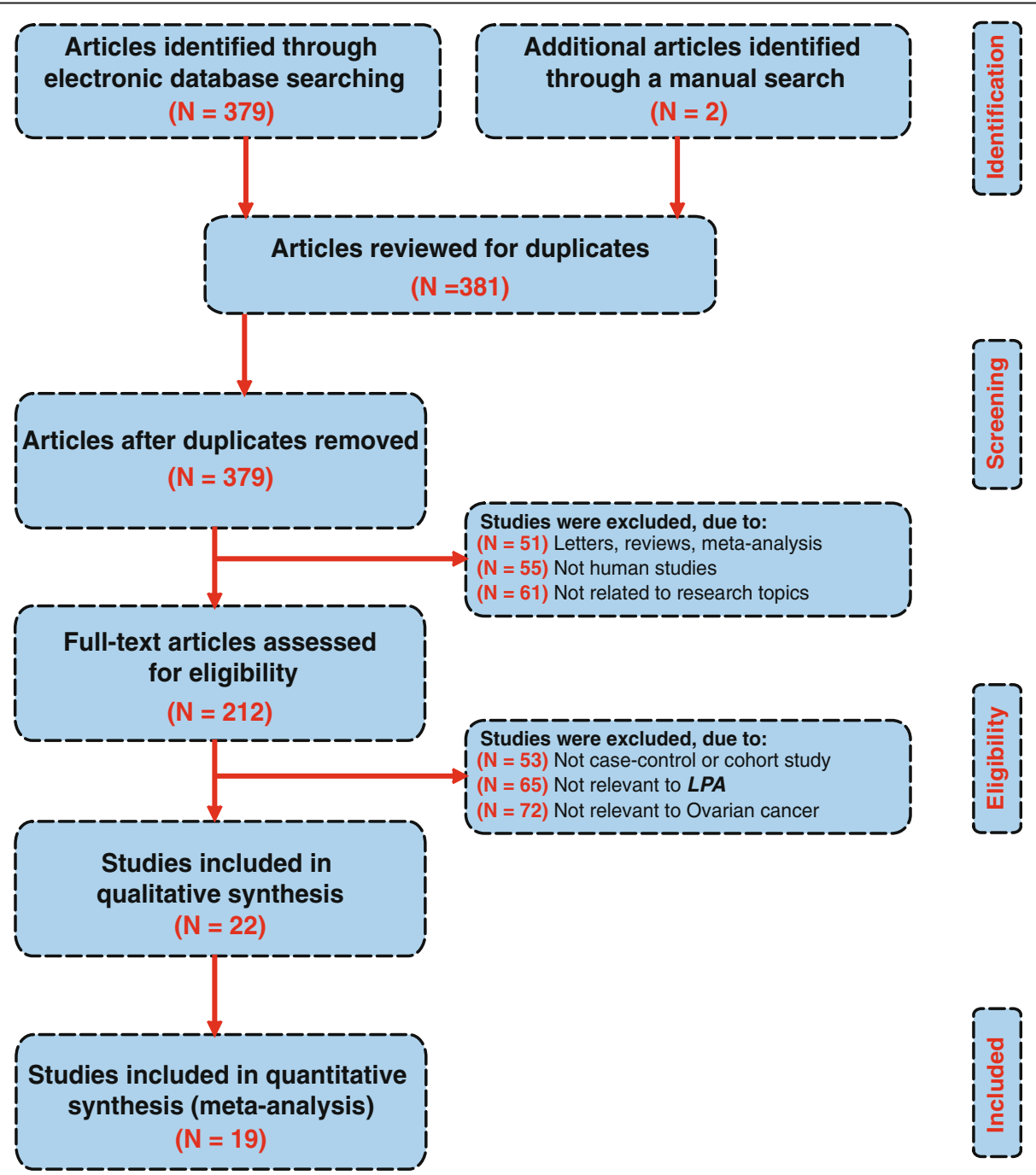

Fig. 1 Flow chart shows study selection procedure. Nineteen studies were included in this meta-analysis 


\section{Materials and methods Literature search}

The following computerized databases were searched electronically (last updated search in May $30^{\text {st }}, 2014$ ) for published studies reporting a correlation between plasma LPA levels and OC: China BioMedicine (CBM), Cochrane Library, PubMed and China National Knowledge Infrastructure (CNKI). The following keywords and search terms were used: ("lysophosphatidic acid" or "MOPA" or "LPA" or "1-oleoyl-lysophosphatidic acid" or "monooleylphosphatidate" or "1-O-oleyllysophosphatidic acid") and ("ovarian neoplasms" or "ovary neoplasms" or "ovary cancers" or "ovarian cancer" or "cancer of ovary" or "ovarian carcinoma" or "ovarian adenocarcinoma" or "ovarian tumor" or "EOC"). The language of publication was not a restriction in our search criteria. Bibliographies of closely related studies were further examined manually to identify additional studies relevant to this meta-analysis.

\section{Study selection}

The inclusion criteria for selection of published studies for this meta-analysis were: (1) OC patients must be confirmed by pathological diagnosis; (2) study design must be case-control studies reporting the correlation between plasma LPA levels and OC; (3) the plasma LPA levels and sample size must be supplied; (4) published studies with full text. If a $50 \%$ identity in study subjects were identified between two extracted studies, only the study with the largest sample size was enrolled. The latest and most complete study was chosen from studies published by same authors.

\section{Data extraction}

Two investigators independently extracted data from the selected studies, based on the pre-determined selection criteria, and any disagreements were resolved by discussion and reexamination. The following information was extracted: first author, publication date, country and ethnicity, study type and design, sample size, sex and age of subjects, detection method for plasma LPA levels, and plasma levels of LPA in OC patients, benign controls and healthy controls.

\section{Quality assessment}

To assess the quality of the selected studies, the two investigators used the criteria outlined in the Critical Appraisal Skill Program (CASP) (http://www.casp-uk.net/\#!casptools-checklists/c18f8). The CASP criteria for casecontrol studies include Section A (CASP01 CASP07), Section B (CASP08 CASP09) and Section C (CASP10 CASP11): clear focus in the study (CASP01); appropriate research questions and pertinent answers to the questions (CASP02); propriety in the case enrollment (CASP03); propriety in the control selection (CASP04); accuracy in the measurement of exposure factors for the least bias (CASP05); controls with other important confounding factors (CASP06); completeness of research results (CASP07); precision research results (CASP08); reliability of research results (CASP09); applicability of research results to the local population (CASP10); coherence of research results to other available evidence (CASP11).

\section{Statistical analysis}

The summary standard mean differences (SMDs) and their $95 \%$ confidence interval $(\mathrm{CI})$ were calculated and $\mathrm{Z}$ test was used to estimate the effect size. The SMDs for plasma LPA levels were aggregated utilizing STATA software, version 12.0 (Stata Corp, College Station, TX, USA) independently by two investigators. If heterogeneity was detected, random-effects model was employed for meta-analysis; otherwise fixed-effects model was adopted. Cochran's Q-statistic was used to evaluate the heterogeneity across the enrolled studies, $P<0.05$ referring to statistical significance. $I^{2}$ test was used to provide further evidence of heterogeneity, with $0 \%$ as no heterogeneity and $100 \%$ as maximal heterogeneity $[16,17]$. If heterogeneity was detected, meta-regression and subgroup

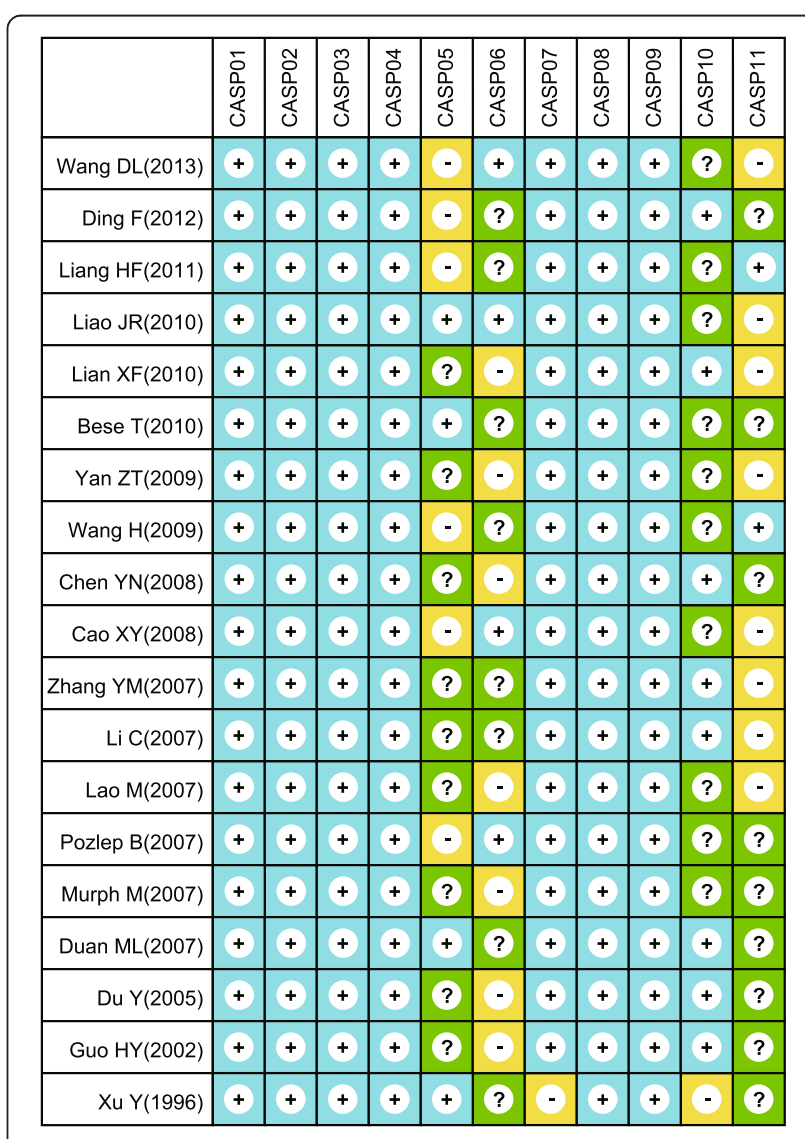

Fig. 2 The methodological quality of eligible studies using critical appraisal skill program criteria (+: Yes; -: No; ? : Unclear) 
analysis based on ethnicity and detection methods were conducted to explore potential influencing factors. The influence of any single study on the overall results was verified by sensitivity analysis. To ensure the accuracy of the results, publication bias was evaluated by constructing a funnel plot, with the symmetry of the funnel plot as evidence of no publication bias and vice versa. Classic fail-safe $\mathrm{N}$ and Egger's linear regression test was used for verifying the results displayed by the funnel plot [18].

\section{Results}

\section{Included studies}

Figure 1 presents the study inclusion process. A total of 381 studies were initially retrieved from database searches. From the retrieved studies, 169 studies were excluded for being duplicates $(n=2)$, letters, reviews or meta-analyses $(n=51)$, non-human studies $(n=55)$, or studies unrelated to the present topic $(n=61)$ and 190 were not for being non-casecontrol studies $(n=53)$, studies irrelevant to LPA $(n=65)$, or studies irrelevant to OC $(n=72)$, and another 3 others studies were removed because they failed to provide did not have the complete data. Finally, 19 case-control studies met the inclusion criteria [11-15, 19-32]. Fig. 2 presents the methodological quality assessment for these 19 studies. All 19 studies reported the correlation between plasma LPA levels and OC and were published between 1996 and 2013. Table 1 lists the demographic information on the OC patients and the baseline characteristics of the studies. Among the 19 studies, 16 studies were performed in Asian populations [China $(n=15)$ and Turkey $(n=1)$ ], and 3 were performed in Caucasians [Slovenia $(n=1)$ and USA $(n=2)$ ]. Collectively, the 19 studies contained a total of 2,520 subjects, with 980 OC patients, 872 benign controls and 668 healthy controls. The plasma LPA levels in patients and controls were detected by ELISA $(n=13)$, bioassay $(n=4)$, Inorganic phosphorus $(n=1)$ and phosphorus determination $(n=1)$.

\section{Meta-analysis of findings}

As shown in Fig. 3, the pooled SMDs revealed that the plasma levels of LPA in OC patients were significantly higher than the benign controls $(\mathrm{SMD}=2.36,95 \% \mathrm{CI}$ : $1.61-3.10, P<0.001)$ and healthy controls $(\mathrm{SMD}=2.32$, $95 \%$ CI: $1.77-2.87, P<0.001)$. The results of subgroup

Table 1 Main characteristics and methodological quality of eligibly studies

\begin{tabular}{|c|c|c|c|c|c|c|c|c|c|c|}
\hline \multirow[t]{2}{*}{ First author } & \multirow[t]{2}{*}{ Year } & \multirow[t]{2}{*}{ Country } & \multirow[t]{2}{*}{ Ethnicity } & \multicolumn{3}{|c|}{ Number } & \multicolumn{3}{|l|}{ Age (years) } & \multirow[t]{2}{*}{ Methods } \\
\hline & & & & Tumor & Benign & Normal & Tumor & Benign & Normal & \\
\hline Wang DL [12] & 2013 & China & Asians & 80 & 40 & 30 & - & - & $34 \sim 67$ & ELISA \\
\hline Ding F [13] & 2013 & China & Asians & 36 & 26 & 20 & 46.7 & 40.5 & 44.5 & Bioassay \\
\hline Liang HF [25] & 2011 & China & Asians & 42 & 408 & 0 & $25 \sim 75$ & & & Bioassay \\
\hline Liao JR-a [24] & 2010 & China & Asians & 8 & 30 & 30 & - & - & - & ELISA \\
\hline Liao JR-b [24] & 2010 & China & Asians & 40 & 30 & 30 & - & - & - & ELISA \\
\hline Lian XF [26] & 2010 & China & Asians & 31 & 40 & 50 & $22 \sim 76$ & $19 \sim 71$ & $20 \sim 71$ & Inorganic phosphorus measurement \\
\hline Bese T [11] & 2010 & Turkey & Asians & 87 & 74 & 50 & - & - & - & Bioassay \\
\hline Yan ZT [21] & 2009 & China & Asians & 76 & 35 & 29 & - & - & - & ELISA \\
\hline Wang $\mathrm{H}[23]$ & 2009 & China & Asians & 30 & 32 & 36 & $28 \sim 68$ & & & ELISA \\
\hline Chen YN [32] & 2008 & China & Asians & 24 & 10 & 10 & 52 & 49 & 50 & Bioassay \\
\hline Cao XY [15] & 2008 & China & Asians & 36 & 36 & 36 & 52.8 & 52.3 & 50.4 & Phosphorus determination method \\
\hline Zhang YM [20] & 2007 & China & Asians & 50 & 44 & 20 & $29 \sim 73$ & & & ELISA \\
\hline Li C-a [27] & 2007 & China & Asians & 60 & 0 & 60 & $27 \sim 65$ & & & ELISA \\
\hline Li C-b [27] & 2007 & China & Asians & 60 & 0 & 60 & $27 \sim 65$ & & & ELISA \\
\hline Lao M [28] & 2007 & China & Asians & 80 & 40 & 40 & $19 \sim 68$ & $19 \sim 65$ & $18 \sim 55$ & ELISA \\
\hline Pozlep B [19] & 2007 & Slovenia & Caucasians & 142 & 0 & 78 & $48 \pm 16.07$ & & 42.2 & ELISA \\
\hline Murph M [14] & 2007 & USA & Caucasians & 26 & 27 & 25 & - & - & - & ELISA \\
\hline Duan ML-a [30] & 2007 & China & Asians & 30 & 30 & 30 & - & - & - & ELISA \\
\hline Duan ML-a [30] & 2007 & China & Asians & 30 & 30 & 30 & - & - & - & ELISA \\
\hline Du Y [31] & 2005 & China & Asians & 37 & 0 & 30 & $53.5 \pm 7.65$ & & $51.6 \pm 6.57$ & ELISA \\
\hline Guo HY-a [29] & 2002 & China & Asians & 16 & 0 & 46 & $56.5 \pm 8.64$ & - & - & ELISA \\
\hline Guo HY-b [29] & 2002 & China & Asians & 15 & 0 & 46 & $56.2 \pm 10.9$ & - & - & ELISA \\
\hline $\mathrm{Xu} Y$ Y [22] & 1996 & USA & Caucasians & 34 & 0 & 48 & - & - & - & ELISA \\
\hline
\end{tabular}


A

LPA

(Ovarian cancer patients VS. Benign controls)

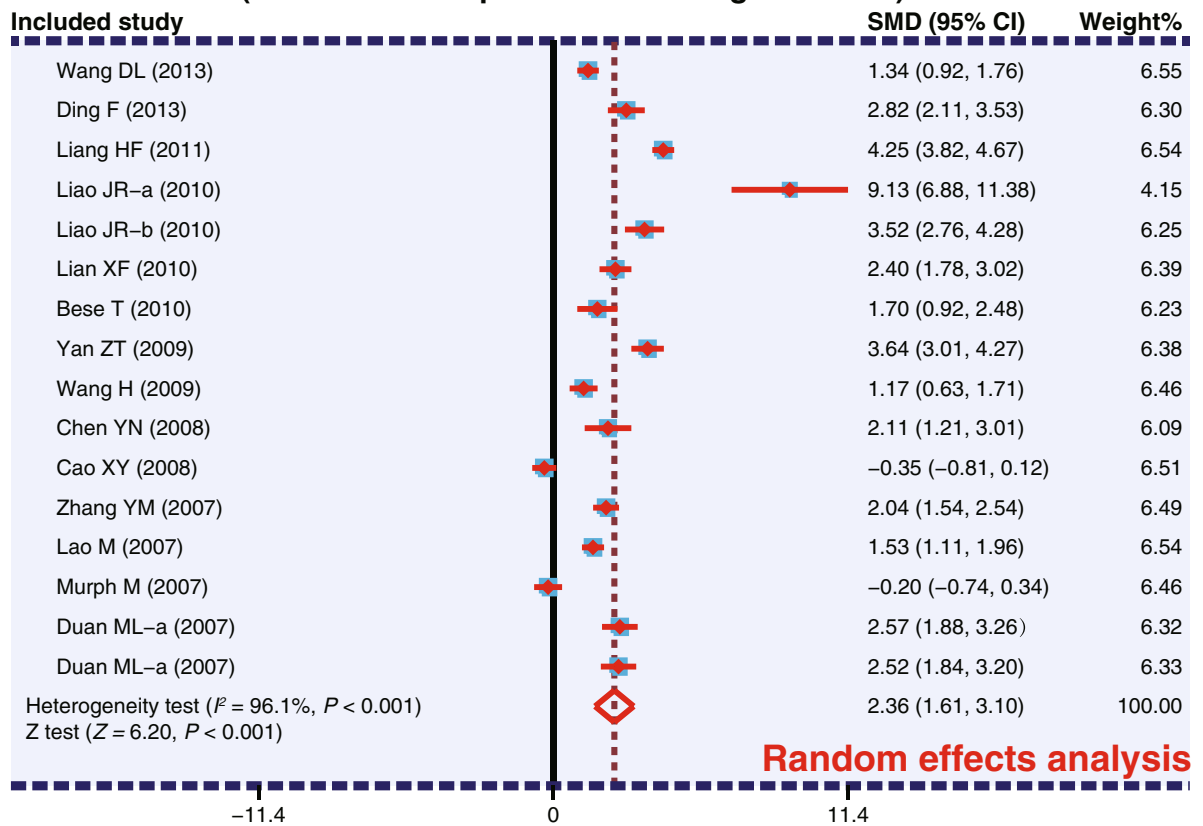

B

LPA

(Ovarian cancer patients VS. Healthy controls)

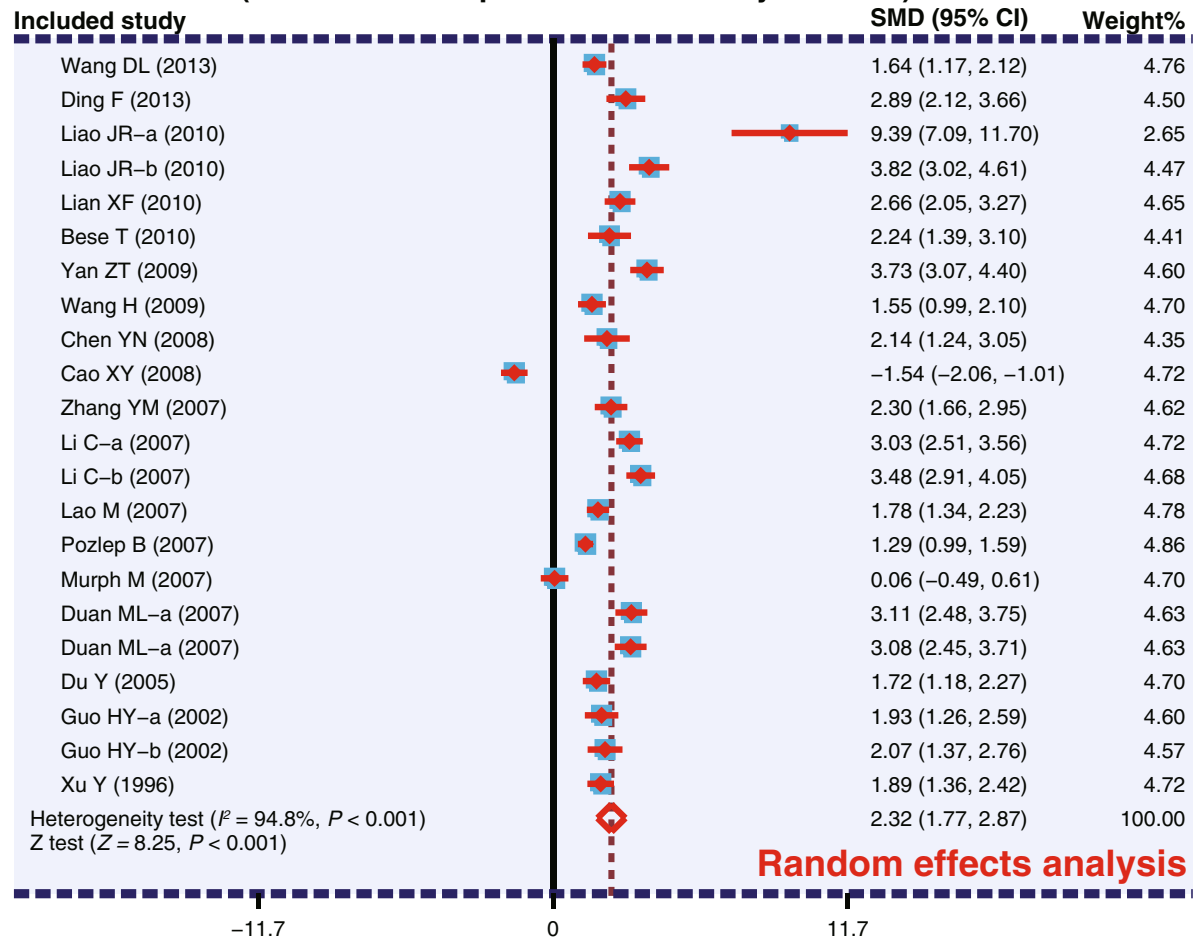

Fig. 3 Forest plots for the relationship between plasma lysophosphatidic acid levels and ovarian cancer (a: Ovarian cancer patients VS. Benign controls; b: Ovarian cancer patients VS. Healthy controls) 
analysis based on ethnicity and detection methods are as follows: plasma LPA levels in OC patients are significantly higher compared to benign controls only among the Asian subjects $(\mathrm{SMD}=2.52,95 \% \mathrm{CI}: 1.79-3.25, P<0.001)$, as quantified by both ELISA (SMD $=2.42,95 \%$ CI: $1.62-3.23$, $P<0.001)$ and non-ELISA methods (SMD $=2.15,95 \% \mathrm{CI}$ : $0.56-3.75, P=0.008$ ). Such a significant difference in plasma LPA levels between OC patients and benign controls was not found in the Caucasian population studied $(P>0.05)$ (as shown in Fig. 4a-b). However, significantly higher plasma LPA levels were found in OC patients, compared to healthy controls, in both Asian (SMD $=2.53,95 \%$ CI: 1.91-3.16, $P$ $<0.001)$ and Caucasian populations $(\mathrm{SMD}=1.09,95 \% \mathrm{CI}$ : $0.20-1.98, P=0.017)$ and in the ELISA subgroup (SMD = 2.47, $95 \%$ CI: $1.95-2.98, P<0.001)$, but not in the nonELISA subgroup $(P>0.05)$ (Fig. 4c-d).

Sensitivity analysis suggested that no single study had an impact on the overall statistical significance. For comparison between $\mathrm{OC}$ patients and benign controls, the constructed funnel plot was symmetrical, suggesting no publication bias, while it was asymmetrical for the comparison between OC patients and healthy controls (Fig. 5).

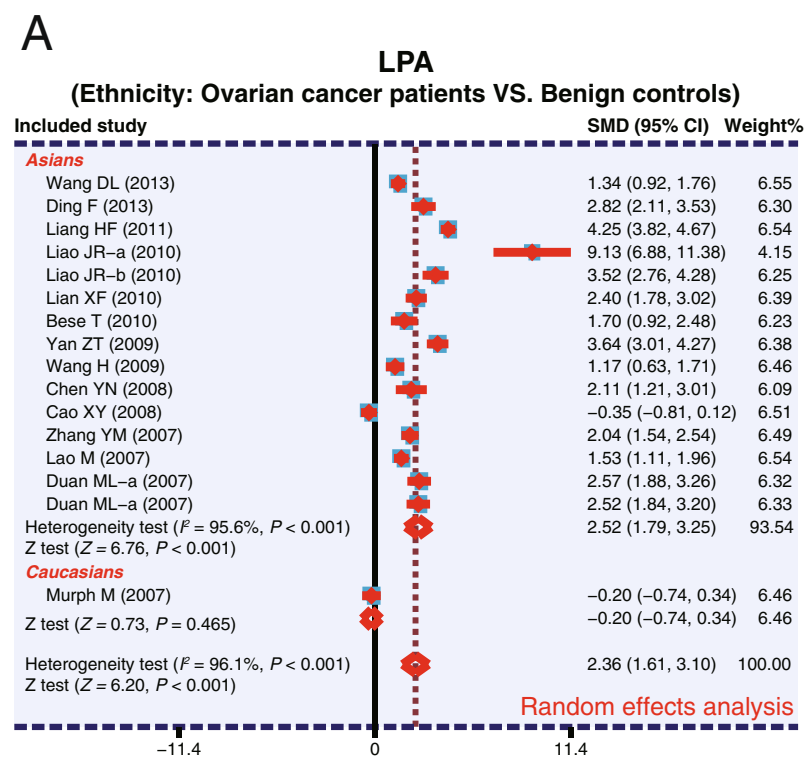

C

LPA

(Ethnicity: Ovarian cancer patients VS. Healthy controls) Included study

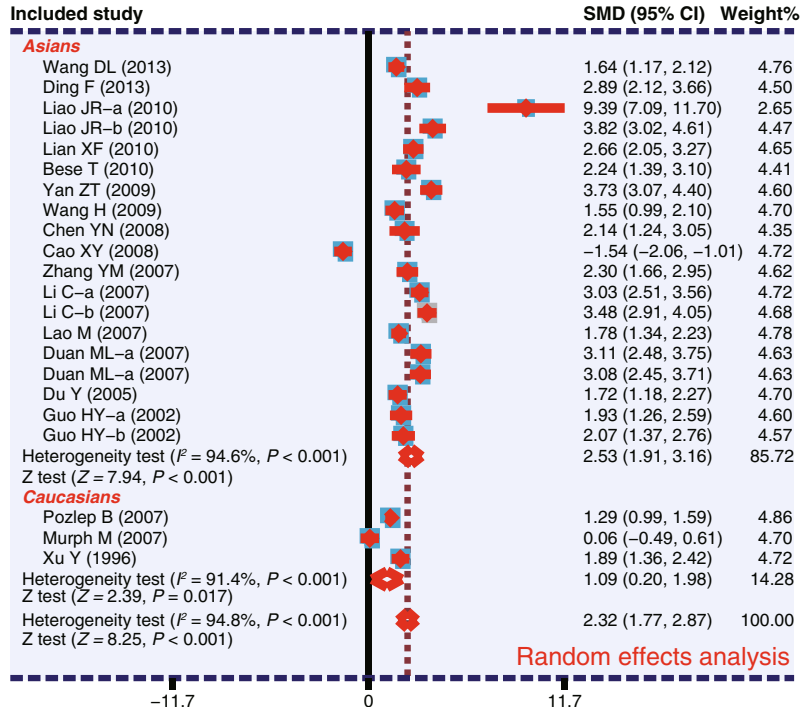

B

(Methods: Ovarian cancer patients VS. Benign controls)

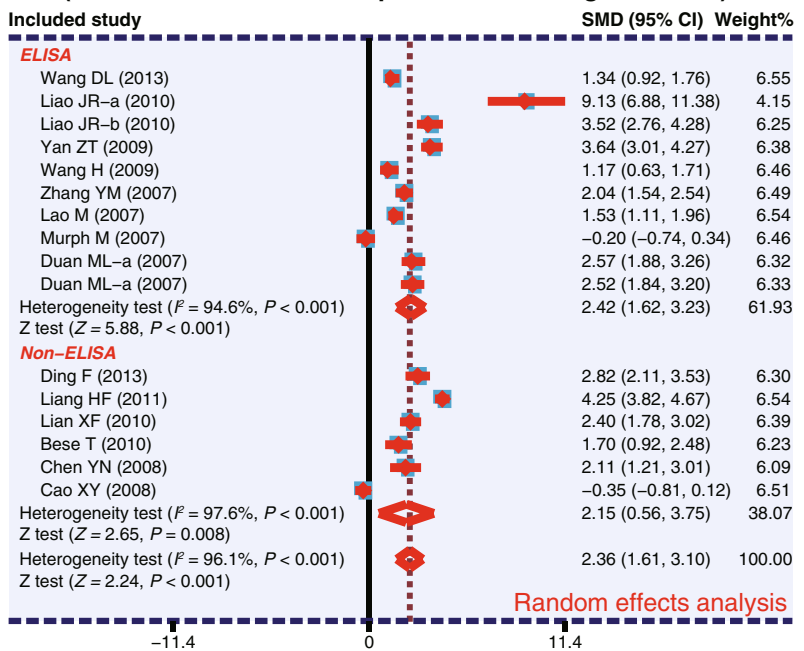

D

LPA

(Methods: Ovarian cancer patients VS. Healthy controls)

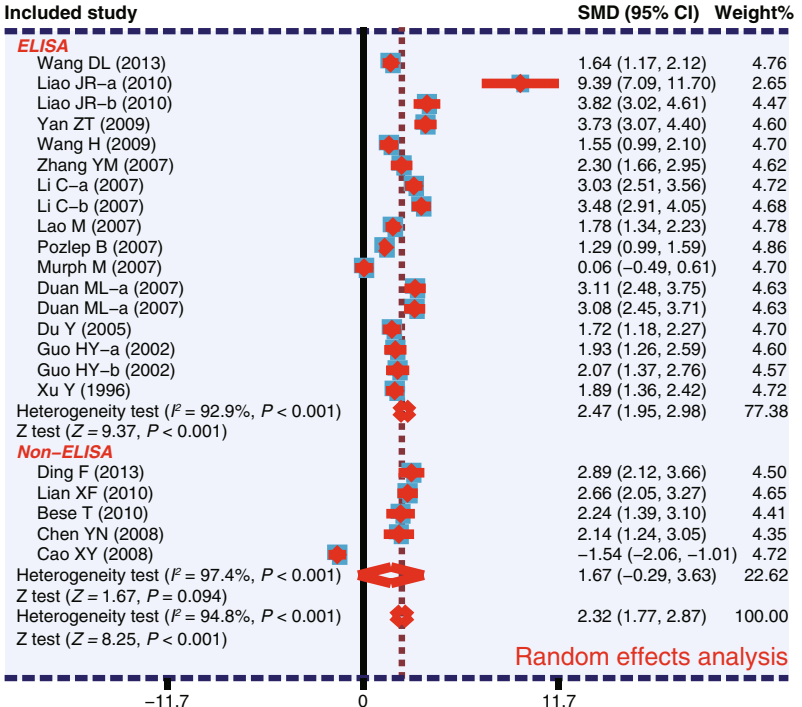

Fig. 4 Subgroup analysis for the relationships between plasma lysophosphatidic acid levels and ovarian cancer (a: Ethnicity: Ovarian cancer patients VS. Benign controls; b: Methods: Ovarian cancer patients VS. Benign controls; c: Ethnicity: Ovarian cancer patients VS. Healthy controls; d: Methods: Ovarian cancer patients VS. Healthy controls) 


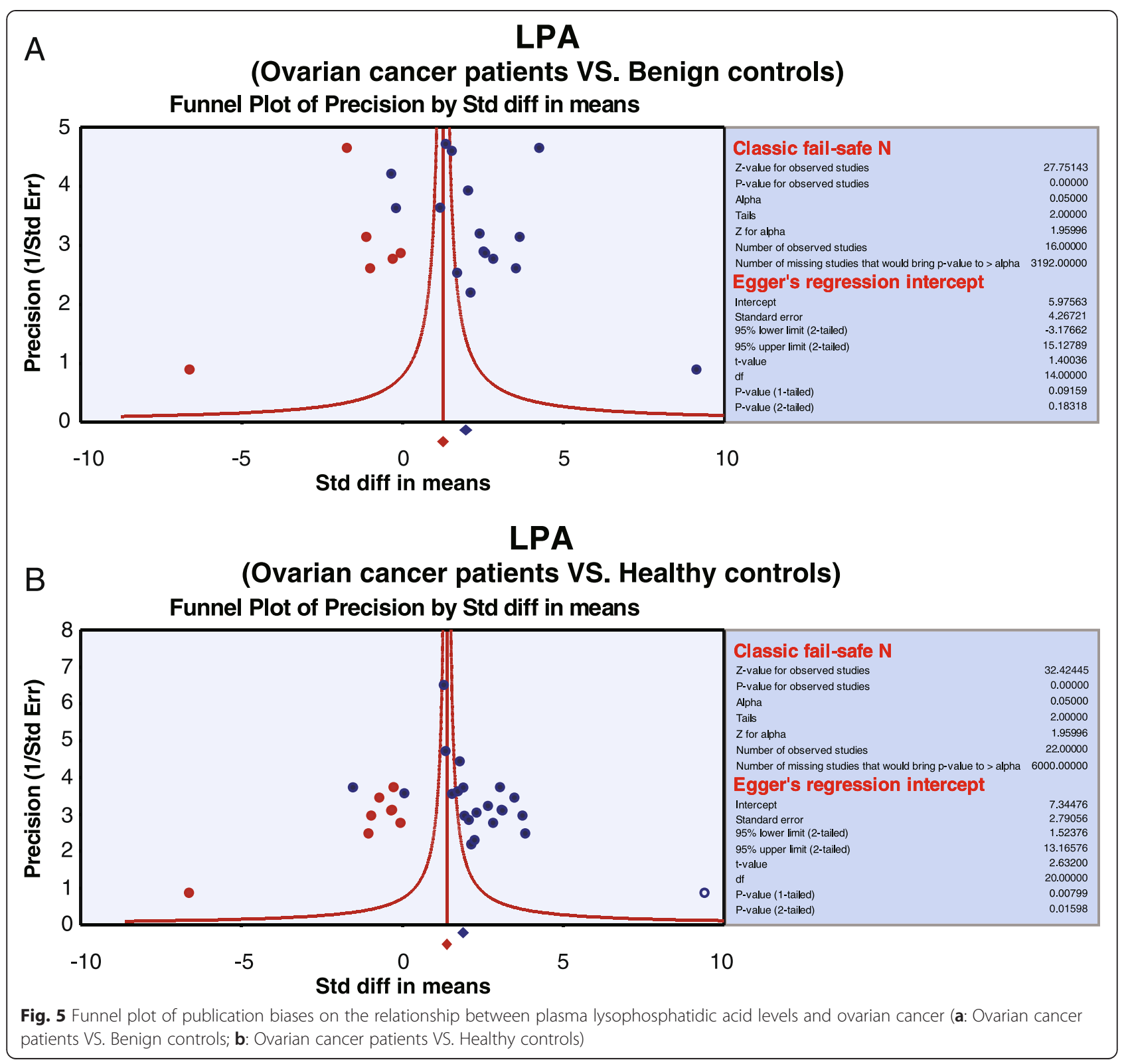

\section{Discussion}

Based on the analysis presented in this study and the data from previous studies, LPA and OC appear to be strongly linked. For example, Hu et al. showed that LPA stimulates ovarian cancer cells by a dual mechanism: (1) LPA indirectly promotes tumor growth by acting as a chemoattractant to endothelial cells, resulting in increased angiogenesis and (2) directly increases the cell proliferation through cyclin D1 activation [33]. Autotaxin (ATX) is the predominant enzyme that produces LPA and ATX transgene expression in mouse mammary glands is sufficient to induce tumors. Conversely, ATX or LPA receptor knockdowns prevent bone metastasis and decrease tumor incidence and progression, respectively, in mouse models of chemically induced carcinogenesis. As such, ATX-LPA signaling in cancer is one of the prominent pathways for tumorigenesis and is a prime target for current therapeutic drug development [9]. Interestingly, contradictory results were obtained by several studies which could not find a clear correlation between LPA and OC. In order to examine this issue closer, we pooled the data from several high-quality published studies and performed a meta-analysis to obtain a better correlation between LPA and OC.

Our meta-analysis clearly showed that the plasma LPA levels in OC patients are significantly higher compared to the benign and healthy controls. Angiogenesis is a key factor for tumor growth and involves VEGF and the activation of VEGF receptors, Flt1 and KDR [19]. OC patients exhibit elevated serum levels of both VEGF and 
LPA [34]. ATX is an autocrine motility factor and a member of the ectonucleotide pyrophosphatase and phosphodiesterase family of enzymes, but also possesses lysophospholipase D activity. This enzymatic activity hydrolyzes lysophosphatidylcholine to generate the potent tumor growth factor and mitogen, LPA $[8,35]$. ATX is highly overexpressed in OC, thus, tumor cells are the major source of LPA production in OC patients [8]. Notably, VEGF signaling was shown to further elevate ATX expression and, thus, OC patients have increased PLD activity and higher LPA plasma levels, compared to healthy controls.

A subgroup analysis was performed to identify factors influencing our study results. The potential factors tested were ethnicity, country and experimental method. Based on the limitations of the data available to us from the selected studies, we focused on ethnicity and detection methods for subgroup analysis. Our subgroup analysis based on ethnicity showed that OC patients from Asian populations exhibited higher plasma LPA levels compared to their benign and healthy counterparts, and the difference was statistically significant. Caucasian OC patients showed significantly higher plasma LPA levels compared to the healthy controls but not the benign counterparts. However, these results may be influenced by the fact that majority of the studies were performed in Asian populations (16 in Asians and 3 in Caucasians). Based on the detection methods, higher plasma LPA levels were observed in OC patients, compared to both benign and healthy controls, using ELISA based methods. The results from the analysis of non-ELISA based methods were largely in agreement with ELISA based results, but a significant correlation between LPA and OC was not observed between the OC patients and healthy controls using non-ELISA methods. This negative result may be due to the small sample size of nonELISA methods. Collectively, our data provide strong evidence that LPA may be involved in OC development or is produced by the tumor and thus may be a tumor marker or target of treatment.

Our study has several significant limitations. Firstly, the dataset used in this study was limited by nineteen prospective trials, which might not be ideal for statistical analysis. Secondly, due to the heterogeneity of the disease, a uniform histopathological diagnosis would have ensured consistent diagnostic standards, which was not the case in the selected studies. Thirdly, some of the studies did not provide complete data and therefore plasma LPA levels at different stages of OC progression could not be compared. Fourthly, sample sizes of OC cases, controls and healthy controls varied widely. Therefore, for a more accurate statistical analysis, a comprehensive dataset and proportionate sample size may be needed. Future studies also must attempt to compare plasma LPA levels at different stages of $\mathrm{OC}$ progression to obtain more thorough disease correlations.

\section{Conclusion}

In summary, a significantly higher plasma LPA levels were observed in OC patients compared to benign controls and healthy controls. Based on our results, we conclude that plasma LPA level is closely correlated to OC and may involve in the development of OC. However, further studies are needed to confirm our findings and explore therapeutic targets within the identified pathway.

\section{Abbreviations}

OC: Ovarian carcinoma; LPA: Lysophosphatidic acid; Edg: Endothelial differentiation gene; NOS: Newcastle-Ottawa scale; SMDs: Standard mean differences; $\mathrm{Cl}$ : Confidence interval.

\section{Competing interests}

The authors declare that they have no competing interests.

\section{Authors' contributions}

$\mathrm{CZ}$ carried out the experiments, $\mathrm{HZ}$ prepared the tables and figures, JZ performed the statistical analysis, YL and WZ wrote the manuscript. HY and LF participated in the interpretation of the data and writing the manuscript. The manuscript received final confirmation from all the authors. All authors read and approved the final manuscript.

\section{Acknowledgments}

This study was funded by the Postdoctoral Science Foundation of China (nos. 2014T70298, 2013M541316, 2013M540256),the training program of Jilin universiy oustanding young teacher project (no. 41908050037). We owe sincere thanks to the excellent reviewers for the great suggestion for my paper.

\section{Author details}

${ }^{1}$ Department of Gynaecology, the First Hospital of Jilin University, Changchun 130041, China. ${ }^{2}$ Center for Reproductive Medicine, Renji Hospital, Shanghai Jiao Tong University School of Medicine, Shanghai, China. ${ }^{3}$ Department of Gynaecology and Obstetrics, the Second Hospital of Jilin University, Ziqiang Street NO. 218, Nanguan District, Changchun 130041, People's Republic of China. ${ }^{4}$ Deparment of Pathology and Pathophysiology, School of Basic Medical Sciences, Changchun 130021, China.

Received: 20 December 2014 Accepted: 30 June 2015

Published online: 15 July 2015

\section{References}

1. Ferlay J, Soerjomataram I, Dikshit R, Eser S, Mathers C, Rebelo M, et al. Cancer incidence and mortality worldwide: sources, methods and major patterns in GLOBOCAN 2012. Int J Cancer. 2015;136:E359-86.

2. Kobayashi E, Ueda Y, Matsuzaki S, Yokoyama T, Kimura T, Yoshino K, et al. Biomarkers for screening, diagnosis, and monitoring of ovarian cancer. Cancer Epidemiol Biomarkers Prev. 2012;21:1902-12.

3. Lowe KA, Chia VM, Taylor A, O'Malley C, Kelsh M, Mohamed M, et al. An international assessment of ovarian cancer incidence and mortality. Gynecol Oncol. 2013;130:107-14.

4. Sedlakova I, Vavrova J, Tosner J, Hanousek L. Lysophosphatidic acid (LPA)-a perspective marker in ovarian cancer. Tumour Biol. 2011;32:311-6.

5. Fang X, Schummer M, Mao M, Yu S, Tabassam FH, Swaby R, et al. Lysophosphatidic acid is a bioactive mediator in ovarian cancer. Biochim Biophys Acta. 2002;1582:257-64.

6. Smicun $Y$, Reierstad S, Wang FQ, Lee C, Fishman DA. S1P regulation of ovarian carcinoma invasiveness. Gynecol Oncol. 2006;103:952-9.

7. Wang FQ, Ariztia EV, Boyd LR, Horton FR, Smicun Y, Hetherington JA, et al. Lysophosphatidic acid (LPA) effects on endometrial carcinoma in vitro proliferation, invasion, and matrix metalloproteinase activity. Gynecol Oncol. 2010;117:88-95.

8. Aoki J. Mechanisms of lysophosphatidic acid production. Semin Cell Dev Biol. 2004;15:477-89.

9. Aoki J, Inoue A, Okudaira S. Two pathways for lysophosphatidic acid production. Biochim Biophys Acta. 1781;2008:513-8. 
10. Luquain C, Singh A, Wang L, Natarajan V, Morris AJ. Role of phospholipase $D$ in agonist-stimulated lysophosphatidic acid synthesis by ovarian cancer cells. J Lipid Res. 2003;44:1963-75.

11. Bese T, Barbaros M, Baykara E, Guralp O, Cengiz S, Demirkiran F, et al. Comparison of total plasma lysophosphatidic acid and serum CA-125 as a tumor marker in the diagnosis and follow-up of patients with epithelial ovarian cancer. J Gynecol Oncol. 2010;21:248-54.

12. Wang $\mathrm{DL}$, Chen $\mathrm{LX}$, Liao $X \mathrm{~L}$, Liao XJ, Wu QH. The relationship of lysophosphatidic acid and MMP-2 in diagnosing epithelial ovarian carcinoma. Proc Clin Med. 2013;22:403-5.

13. Ding F, Niu B. Clinical significance of detecting lysophosphatidic acid and vascular endothelial growth factor in patients with epithelial ovarian carcinoma. Practical J Med Pharmacy. 2013;30:775-7.

14. Murph M, Tanaka T, Pang J, Felix E, Liu S, Trost R, et al. Liquid chromatography mass spectrometry for quantifying plasma lysophospholipids: potential biomarkers for cancer diagnosis. Methods Enzymol. 2007;433:1-25.

15. Cao XY. The applicable value of combined detection of LPA, CA1 25 and AFP in the early diagnosis of ovarian cancer. Laboratory Med Clinic. 2008:5:1430-1.

16. Jackson D, White IR, Riley RD. Quantifying the impact of between-study heterogeneity in multivariate meta-analyses. Stat Med. 2012;31:3805-20.

17. Peters JL, Sutton AJ, Jones DR, Abrams KR, Rushton L. Comparison of two methods to detect publication bias in meta-analysis. JAMA. 2006;295:676-80.

18. Zintzaras E, loannidis JP. HEGESMA: genome search meta-analysis and heterogeneity testing. Bioinformatics. 2005;21:3672-3.

19. Pozlep B, Meleh M, Kobal B, Verdenik I, Osredkar J, Kralj LZ, et al. Use of lysophosphatidic acid in the management of benign and malignant ovarian tumors. Eur J Gynaecol Oncol. 2007;28:394-9.

20. Zhang YM, Zhang XN. Expression of XIAP, Survivin, LPA and CA12 5 in human epithelial ovarian carcinoma. Journal of Shandong University (Health Science). 2007:45:317-21.

21. Yan ZT, Huang N, Zhao ML, Pang AP. Clinical Values of serum CA125 and LPA determination in early diagnosis of ovarian cancer. Chinese Journal of Primary Medicine and Pharmacy. 2009;16:1993-4.

22. Xu Y, Shen Z, Wiper DW, Wu M, Morton RE, Elson P, et al. Lysophosphatidic acid as a potential biomarker for ovarian and other gynecologic cancers. JAMA. 1998;280:719-23.

23. Wang $H$, Chen DZ. Study on the diagnostic Value of Plasm a Lysopho sphatidic Acid (LPA) Level Determination in Patients with Ovarian Carcinoma. Journal of Radioimmunology. 2008;21:355-7.

24. Liao JR, Zong JH, LV YY. Detection of Plasma lysophosphatidic Acid (LPA) in Epithelial Ovarian Tumor Patients. Inner Mongolia Medical Journal. 2010;42:1295-6.

25. Liang HF, Zhang H, Ma XY. Comparative analysis of the value of lysophosphatidic acid and CA125 in the diagnosis of ovarian cancer. Clinical Medicine of China. 2011;27:348-50.

26. Lian XF, Li XL, Zhan HL, Sun F. The diagnostic value when lysophospholipids acid, epididymis protein 4 used in early ovarian cancer's detection. International Medicine and Health Guidance News. 2010;16:2778-81.

27. Li C, Fang SH, Chen W. The diagnostic value of sol uble P185 in ovarian cancer. Chinese Journal of Laboratory Diagnosis. 2007;11:1238-9.

28. Lao M, Pan ZM, Huang WC, Huang LS, Zhu B, Zhao HL, et al. The value of plasma lysophosphatidic acid measurement in the diagnosis of gynecology tumor. The Practical Journal of Cancer. 2007;22:347-9.

29. Guo HY, Han JS, Wu QZ, Yang CS. A study of diagnostic value of plasma lysophosphatidic acid for ovarian epithelial cancer. Chinese Journal of Clinical Obstetrics and Gynecology. 2002;03:36-8.

30. Duan ML, Wu F. The diagnostic value of secretory phospholipase A2 and lysophosphatic acid for ovarian cancer. Chinese Journal of Laboratory Medicine. 2005;28:622-4.

31. Du Y. Study on correlation between diagnosis of epithlial ovarian cancer and plasma lysophosphatidic acid. Zhejiang Medical Journal. 2005;27:827-8.

32. Chen YN, Tao M, Tu FP, Zuo Y, Lu WD. LPA, IL-6 and IL-8 levels in the plasma of patients with epithelial ovarian carcinoma. The Practical Journal of Cancer. 2008;23:580-2.

33. Hu YL, Albanese C, Pestell RG, Jaffe RB. Dual mechanisms for lysophosphatidic acid stimulation of human ovarian carcinoma cells. J Natl Cancer Inst. 2003;95:733-40.
34. Hu YL, Tee MK, Goetzl EJ, Auersperg N, Mills GB, Ferrara N, et al. Lysophosphatidic acid induction of vascular endothelial growth factor expression in human ovarian cancer cells. J Natl Cancer Inst. 2001;93:762-8.

35. Nakamura K, Igarashi K, Ohkawa R, Yokota H, Masuda A, Nakagawa S, et al. Serum autotaxin is not a useful biomarker for ovarian cancer. Lipids. 2012:47:927-30.

\section{Submit your next manuscript to BioMed Central and take full advantage of:}

- Convenient online submission

- Thorough peer review

- No space constraints or color figure charges

- Immediate publication on acceptance

- Inclusion in PubMed, CAS, Scopus and Google Scholar

- Research which is freely available for redistribution 